\title{
Study and Development of Fuel Adulteration Detection System
}

\author{
Harsh Mahajan ${ }^{1}$, Geetesh Mokhare ${ }^{2}$, Ankita H. Harkare ${ }^{3}$, \\ Deepak G. Khushalani ${ }^{4}$, B.A. Neole ${ }^{5}$ and Rakshal Agrawal ${ }^{6}$ \\ ${ }^{1}$ Research Intern, Shri Ramdeobaba College of Engineering and Management, \\ Nagpur, India, Department of Electronics and Communication Engineering \\ ${ }^{2}$ Research Intern, Shri Ramdeobaba College of Engineering and Management, \\ Nagpur, India, Department of Electronics and Communication Engineering \\ ${ }^{3}$ Assistant Professor, Shri Ramdeobaba College of Engineering and Management, \\ Nagpur, India, Department of Electronics and Communication Engineering \\ ${ }^{4}$ Assistant Professor, Shri Ramdeobaba College of Engineering and Management, \\ Nagpur, India, Department of Electronics and Communication Engineering \\ ${ }^{5}$ Assistant Professor, Shri Ramdeobaba College of Engineering and Management, \\ Nagpur, India, Department of Electronics and Communication Engineering \\ ${ }^{6}$ Assistant Professor, Shri Ramdeobaba College of Engineering and \\ Management, Nagpur, India, Department of Industrial Engineering
}

\section{ABSTRACT}

Over the years, the rise in the fuel prices has lead the adulteration market to develop new techniques for blending the inferior adulterants into the highly taxed petroleum products. Despite of various adulteration monitoring techniques existing in place, this illegal practice of profit making by using subsidized fuels as adulterants still persists. The emission from consumption of such adulterated fuels has contributed to the global Air pollution and various health hazards linked to it. This calls for the ease and precision of monitoring techniques. This study reviews the use of emerging fields of technology like microfluidics, metamaterial, microfabrication and fiber optics to develop Adulteration detection systems for Diesel which provides higher sensitivity and portability as compared to the existing lab based methods. Also, experimentation results measuring density of the fuel suggests adulteration above $16.66 \%$ is unacceptable.

KEY WORDS: ADULTERATION, DENSITY, FUEL, MICROFLUIDICS.

\section{ARTICLE INFORMATION}

*Corresponding Author: mahajanh@rknec.edu

Received 14th Oct 2020 Accepted after revision 29th Dec 2020

Print ISSN: 0974-6455 Online ISSN: 2321-4007 CODEN: BBRCBA

Thomson Reuters ISI Web of Science Clarivate Analytics USA and Crossref Indexed Journal

\section{Clarivate
Analytics}

NAAS Journal Score 2020 (4.31)

A Society of Science and Nature Publication,

Bhopal India 2020. All rights reserved.

Online Contents Available at: http//www.bbrc.in/

Doi: $h t t p: / / d x$.doi.org/10.21786/bbrc/13.14/104

\section{INTRODUCTION}

Adulteration is the introduction of an inferior standard foreign substance into a higher-level substance which demeans the overall quality and devalues the product. This practise is unlawful and unethical. After the addition of such materials the final product doesn't meet the legal standards and distribution of which makes the whole process illegal. According to the Forensic guide for Criminal Investigators by NICFS, if the Petrol or High 
Speed Diesel oil (HSDO) fails to fulfil the requirements of the Bureau of Indian Standards Specification no. IS 2796 and IS no. 1460, then it is considered as adulterated. Such products have health as well environmental hazards. The emission from consumption of such contaminated fuel causes mixing of carcinogenic pollutants in the air leading to the various ailments and degradation of air. It also affects the engines, deteriorating their performance and life as explained by Boadu et al.

According to a recent report published in Hindustan times, out of the overall Indian refined fuel sale, diesel consumption occupies $40 \%$ of the total. As classified by the Ministry of Petroleum And Natural Gas, Diesel in it's two forms, i.e. High Speed Diesel oil (HSDO) and LD0 (Light Diesel Oil) showed a growth of $4.4 \%$ and $21.7 \%$ respectively in their consumption making a total volume of 84.126 MMT ( 83.528 TMT HSDO + 598 TMT LDO). This high amount of the consumption gives rise to the economic opportunities for adulteration market as one of the major contributors towards loss of the government's revenue as explained by Economics \&t Statistics Division.

Table 1. List of Adulterants Found in Diesel (HSDO)

\begin{tabular}{|l|c|c|}
\hline Sr No. & Substance & Source \\
\hline 1. & Kerosene & Marketers \\
\hline 2. & MT0 & Refineries \\
\hline 3. & C9 Raffinate & Petrochemicals \\
\hline 4. & Iomex & NA \\
\hline 5. & Aromex & Digboi \\
\hline
\end{tabular}

Tabel 2. ASTM Test Methods for Monitoring Fuel Adulteration

\begin{tabular}{|l|c|}
\hline Tested Parameters & Tests \\
\hline Cetane Number (Ignition Quality) & ASTM D613 \\
\hline Flash Point & ASTM D93 \\
\hline Density & ASTIM D1298 \\
\hline Kinematic Viscosity & ASTM D445 \\
\hline Distilation & ASTM D86 \\
\hline
\end{tabular}

The challenge while testing for the adulterations in fuels is that the adulterants that are being searched are already present in them in their consumed form. There is a range in which their presence is permitted. Instead of the presence, the composition of the adulterated fuel is checked and the results are drawn out by observing the percentage of that adulterant as explained by Bhanu Prasad et al. The existing methodologies that are being used are bulkier and lab based. The tests and standards suggested by The American Society for Testing and Materials (ASTM) are used globally. Some other tests are Paper Filter Test (As suggested by NICFS), Density Measurement (Used mostly in practise). Some of the physico-chemical methods for testing the adulteration are mentioned in Table 2. All these techniques, being lab based, are time demanding and they need trained personals to carry them out.

Moreover, these methods are limited to only the detection. This calls for testing techniques that are fast and equipments that are portable. Multidisciplinary fields like Microfluidics to develop the Lab on Chip solutions to overcome the disadvantages of the lab based testing techniques. Methods involving application of MEMS have also been designed.PDMS moulding techniques have been used to the advantage of PDMS being a low cost material and ease of fabricating micro channels out of it. Rapid bonding along with advantageous optical properties make it a suitable material for prototypes and testing microfluidic systems as suggested by Byung-Ho Jo et al.

\section{MATERIAL AND METHODS}

Various methods have been suggested in recent times for adulteration detection. Some of the methods are discussed here for getting an insight into the suggested topic of research.

I.UItrasonic Radiation: Measuring the speed of the ultrasound through fluids is used as a detection method where Concentration analyzer is used to send ultrasonic pulses through the fluid as medium. Firstly, Diesel is taken as the medium followed by the samples of it mixed with kerosene in known ratios. The observed speed of the sound is then converted into the corresponding adulteration percentage as explained by R.K. Sharma et al. For analyzing the adulteration, the mathematics used to calculate the percentage of adulteration from the observed speed of sound through the Analyzer is given by South Asia Urban Air Quality Management Briefing Note no.7:

$$
\begin{aligned}
& \mathrm{X}=0.0072^{*} \mathrm{~V}-20.397^{*} \mathrm{~V}+14441 \\
& \text { where, } \\
& \mathrm{X}=\text { Percent of Adulteration (by volume), and } \\
& \mathrm{V}=\text { Speed of sound in } \mathrm{m} / \mathrm{sec}
\end{aligned}
$$

R.K. Sharma et al. used pulse echo method to detect the speed of ultrasound in the samples. Figure 1 and 2 suggests the changes in speed of sound and Density of mixture due to increased values of adulteration in the actual fuel. This concept of changes in density of the mixture will be utilized in our experimental set up as well. Though viscosity was not measured but its effect on the speed of sound formed the basis of the experimentation. The test equipment showed precise results and was portable enabling the field testing. Viscosity measurement will be included in the experimental setup suggested by us.

II. Fiber Optics: An optical fiber with a small length of absorbing cladding removed is inserted into a glass container such that the length of the removed cladding is exposed to the test fluid in the container. 
The experiment used the concept of refractometry for the experiment. Sukhdev Roy suggested sending a laser light thorough the optical fiber and the difference in the power transmitted and received was observed. Using the power, the absorbance of the medium was found out and the samples are compared on the basis of the calculated absorption coefficient. Figure 3 defines the suggested experiment setup where the sensitivity of the test is a variable and is directly proportional to the length of the exposed region of the fiber (Length for which the cladding is removed). Till $20 \%$ of the adulteration, the normalized power decreases and then increases with the increase in the adulteration. The test concluded that addition of kerosene beyond 20\% volume by volume, the absorbance of the fuel increases. The experiment gave the variation of bulk absorbance, another physical property on the basis of which the adulterated fuel sample can be monitored. Yet it is not able to tell the extent of adulteration.

Figure 1: Speed of sound in Deisel+Kerosene Mixture

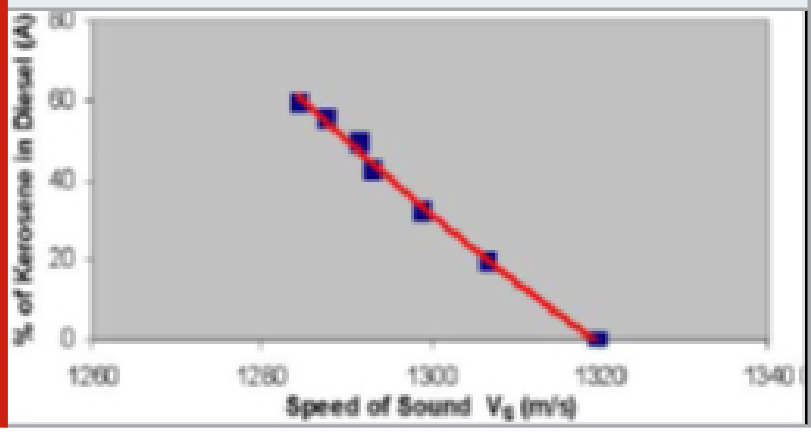

Figure 2: Density of Deisel+Kerosene Mixture

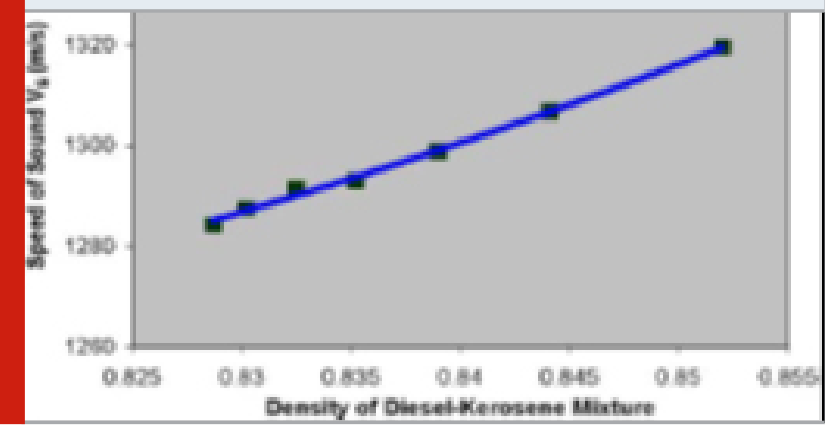

Figure 3: Schematic diagram of the experimental set-up.

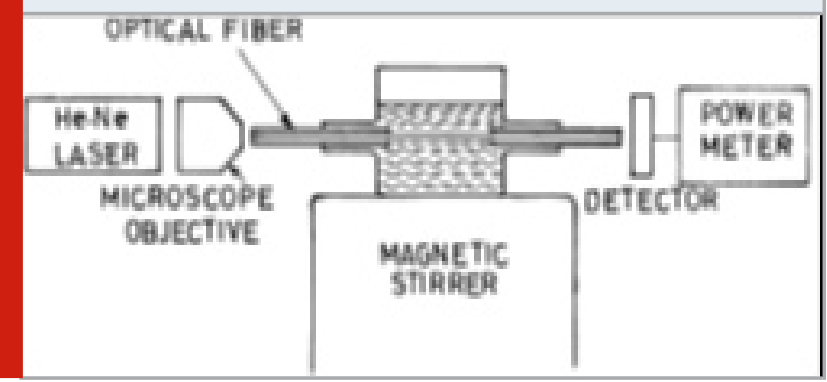

III. Lab on Chip Solutions using MEMS and Microfluidics: Lab on chip devices provide miniaturised and more precise solutions to the lab based experimentations for measurement or processing techniques. Microfluidics incorporates the study of fluidic behaviour at a micro level. At the micro stage, the processing or manipulation of material gets convenient. Such devices give in physical as well as technological advantages. The concept of microfluidics provides a technological liberty as there are numerous analytical and processing techniques for analysis the test samples at a micro level. The Y-channel suggested by Ankita H. Harkare will be utilized for developing the microchannel for sample collection.

Figure 4: (a) Y-shaped channel (b) Y-shaped serpentine channel (c) Y-shaped channel with obstacles.

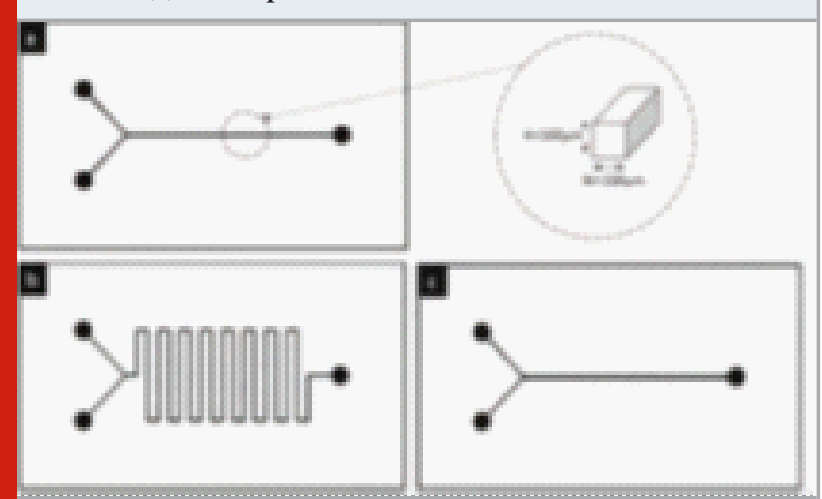

Figure 5: Fabricated Microfluidic chip

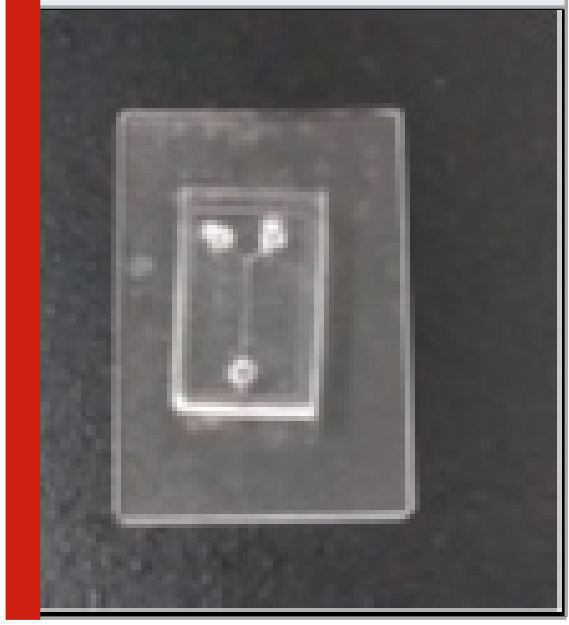

As suggested by Mary Salve et al., Image processing can also be an effective approach for developing a readout technique. One such method can be texture analysis of samples captured by camera to analyse the uniformity of texture and using stochastic processes to compute the adulteration. These can be detected very effectively using the methods suggested by Purva patil et al. Here blurring aspect is highlighted while preserving the sharpness and continuity of edges. The noise is removed by averaging and blurring of images at the edges is minimized. Here, sudden transitions in edges are accurately recognized 
for visual appeal. Image processing can be employed to examine the micro test samples in microfluidic devices. One such technique as suggested uses PDMS micro channel to perform colorimetric detection methodology to detect detergents in milk samples.

\section{Proposed Design and Experimentation:}

I. Microfluidic Channel: The design focuses on developing a Y shaped Microfluidic Hydrometer. Such micro channels cater a better platform for the quantitative analysis. The Microfluidic Hydrometer focuses on detecting the density of the introduced fuel and this will be compared with the Look up table to detect Fuel adulteration in percententages. Using the studies suggested in above methods an effective experimentation setup is suggested which will lead to portable device which can be carried along by the user in order to detect adulteration in fuel.

Figure 6: Block Diagram of Experimentation SetUp

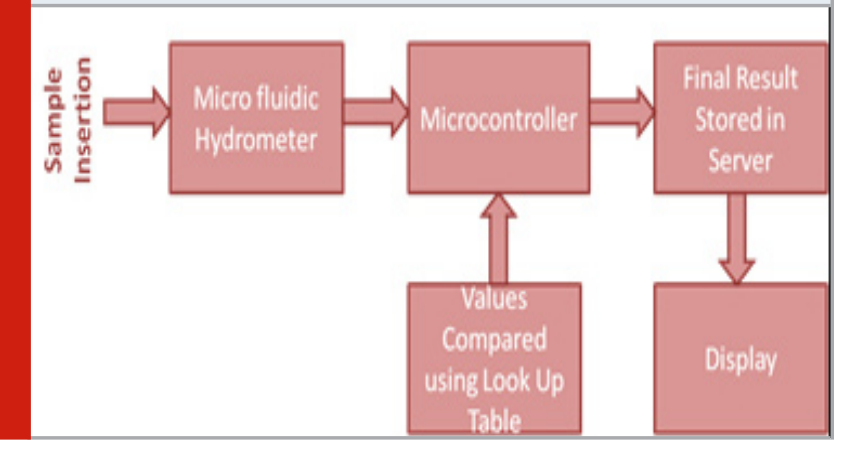

Table 3. Experimentation Results

\begin{tabular}{|c|c|c|c|}
\hline S.N. & $\begin{array}{c}\text { Volume/ } \\
\text { Adulterated } \\
\text { Sample } \\
\text { Mixture }\end{array}$ & \% Adulteration & $\begin{array}{c}\text { Density } \\
\text { (gm/ml) }\end{array}$ \\
\hline 1 & $210 / 0$ & $0-1 \%$ & 1.15 \\
\hline 2 & $200 / 10$ & $5-10 \%$ & 1.55 \\
\hline 3 & $180 / 30$ & $10-16.66 \%$ & 1.16 \\
\hline 4 & $170 / 34$ & $18-25 \%$ & 1.171 \\
\hline 5 & $150 / 60$ & $25-50 \%$ & 1.18 \\
\hline 6 & $120 / 90$ & Above $50 \%$ & 1.182 \\
\hline
\end{tabular}

II. Sample Preparation: The sample mixtures were prepared using HPCL Diesel and Kerosene from the local vendor. Total sample were prepared by introducing kerosene in diesel by volume. The percentage adulterated sample was then used for measuring the density (gm/ $\mathrm{ml})$.

III. Experiment Setup: The experimentation set up is shown in the block Diagram Shown in Figure 6 where the readout setup with require a lookup table which needs to be formulated using experimentation results. The deployment of microcontroller along with RTOS will make it easy to check the adulteration using a handy portable device.The sample experimentation is listed in Table 3. Experimentation results for the density of samples using hydrometer for the development of look up table.

Observations based on the readings suggest that the density of the sample went on increasing with the increase in percentage of kerosene in the sample. The Adulteration of Diesel with Kerosene above 16.66\% volume by volume of the sample is not accepted according to the regulations. These readings will be fed in the system as lookup table which will be utilized to check the samples in micro volumes. However, these readings will still have errors and the quality of diesel might vary for different companies. Hence, in order to make a full proof system it also becomes important to include quality improvement techniques such as Six Sigma to ensure better results. Systematic analysis of data along with the process parameter will be completed which will enable us to focus on the correct identification of error and gradually the errors will be minimized to ensure quality improvement after testing as suggested by Sharma et al.

\section{CONCLUSION}

This review suggests an operative portable testing mechanism monitor the extent of adulteration by determining the density of contaminated test samples and comparing them with that of look up table (According to the standard regulations). The device is designed in a manner which makes it reusable with the same accuracy with each test. Due to the use of RTOS, the device can be recalibrated in accordance with the fluid to be tested which makes the application versatile. Batch production would certainly reduce the cost and the device can then be deployed for the application.

\section{ACKNOWLEDGEMENTS}

We acknowledge Dr. Jayu Kalambe, Co-ordinator, Center of Excellence, Microsystems, RCOEM, Nagpur for making the facilities readily available for use and experimentations.

\section{REFERENCES}

"South Asia Urban Air Quality Management Briefing Note no.7”, July 2002

Ankita H. Harkare, "Analytical Study for Development of Fuel Adulteration Detection System”, Helix Vol. 8(6): 4327- 4333 (2018)

Bhanu Prasad Vempatapu and Pankaj K. Kanaujia "Monitoring petroleum fuel adulteration: A review of analytical methods", Trends in Analytical Chemistry 92 (2017) 1e11

Boadu, K0, "Effects of Adulteration on Diesel Oil with Kerosene Fuel In Ghana”, J.Appl. Sci. Environ. Manage. Vol. 23(7) 1195-1200 (2019)

Byung-Ho Jo, Linda M. Van Lerberghe, Kathleen M. Motsegood, and David J. Beebe, Member, IEEE, 
"Three-Dimensional Micro-Channel Fabrication in Polydimethylsiloxane (PDMS) Elastomer", Journal of Microelectromechanical Systems, Vol. 9, No. 1, March 2000

Economics \&t Statistics Division, "Indian Petroleum \&t Natural Gas Statistics 2018-19”, Ministry of Petroleum and Natural Gas, India

Erick A. Massawe, Hassan kilavo and Anael Sam, "Fuel Adulteration in Tanzania and its Consequences: An overview"; Research Journal in Engineering and Applied Sciences, ISSN:2276-8467, Pg 281-284

Juhyuk Park, Young Seok Song, "Laminar flow manipulators”, Extreme Mechanics Letters, 2020.

Mary Salve, Sakshi Rana, Gurushree Dindorkar, Prakash Rewatkar, and Jayu Kalambe, "Development of Microfluidics-Based Quantitative Adulteration Detection Platform", Sensor Letters, Vol. 16, 1-5, 2018.

NIFSC, A Forensic Guide for Crime Investigators, Chapter 11- Petroleum and Adulteration.

Purva S. Patil, Bhumika A. Neole, Kishor Bhurchandi,
"Performance Evaluation of A New Local Edge Profile Preservation Based Denoising Algorithm“;IEEE International Conference on Signal and Image Processing Applications (IEEE ICSIPA 2017), Malaysia,September 12-14, 2017

R. A. Kishore Nadkarni "Guide to ASTM Test Methods for the Analysis of Petroleum Products and Lubricants", ASTM International, 100 Barr Harbor Drive, PO Box C700, West Conshohocken, PA 19428-2959 (2007)

R.K. Sharma and Anil Kumar Gupta, "Detection/ Estimation of Adulteration in Gasoline and Diesel using Ultrasonics", in Second International Conference on Industrial and Information Systems, ICIIS 2007, 8 - 11 August 2007, Sri Lanka

Sharma, Sandhir "Application of Six Sigma in Electronics Industry"; International Journal of Engineering Science and Innovative Technology, Vol2, 2013

Sukhdev Roy, "Fiber optic sensor for determining adulteration of petrol and diesel by kerosene", Sensors and Actuators, an Elsevier Journal, 1999 